\title{
Dorsal-to-Ventral Shift in Midbrain Dopaminergic Projections and Increased Thalamic/Raphe Serotonergic Function in Early Parkinson Disease
}

\author{
Juho Joutsa $^{1-3}$, Jarkko Johansson ${ }^{2}$, Marko Seppänen ${ }^{4}$, Tommi Noponen ${ }^{4}$, and Valtteri Kaasinen ${ }^{1,2}$ \\ ${ }^{I}$ Turku PET Centre, Turku University Hospital and University of Turku, Turku, Finland; ${ }^{2}$ Division of Clinical Neurosciences, Turku \\ University Hospital and University of Turku, Turku, Finland; ${ }^{3}$ Department of Clinical Neurophysiology, Turku University Hospital \\ and University of Turku, Turku, Finland; and ${ }^{4}$ Department of Clinical Physiology and Nuclear Medicine, Turku University Hospital \\ and University of Turku, Turku, Finland
}

\begin{abstract}
Loss of nigrostriatal neurons leading to dopamine depletion in the dorsal striatum is the pathologic hallmark of Parkinson disease contributing to the primary motor symptoms of the disease. However, Parkinson pathology is more widespread in the brain, affecting also other dopaminergic pathways and neurotransmitter systems, but these changes are less well characterized. This study aimed to investigate the mesencephalic striatal and extrastriatal dopaminergic projections together with extrastriatal serotonin transporter binding in Parkinson disease. Methods: Two hundred sixteen patients with Parkinson disease and 204 control patients (patients without neurodegenerative parkinsonism syndromes and normal SPECT imaging) were investigated with SPECT using the dopamine/ serotonin transporter ligand ${ }^{123} \mid-N$ - $\omega$-fluoropropyl-2 $\beta$-carbomethoxy$3 \beta$-(4-iodophenyl)nortropane ( ${ }^{123}$-FP-CIT) in the clinical setting. The group differences and midbrain correlations were analyzed voxel by voxel over the entire brain. Results: We found that Parkinson patients had lower ${ }^{123}$-FP-CIT uptake in the striatum and ventral midbrain but higher uptake in the thalamus and raphe nuclei than control patients. In patients with Parkinson disease, the correlation of the midbrain tracer uptake was shifted from the putamen to widespread corticolimbic areas. All findings were highly significant at the voxel level familywise error-corrected $P$ value of less than 0.05 . Conclusion: Our findings show that Parkinson disease is associated not only with the degeneration of the nigrostriatal dopamine neurotransmission, but also with a parallel shift toward mesolimbic and mesocortical function. Furthermore, Parkinson disease patients seem to have upregulation of brain serotonin transporter function at the early phase of the disease.
\end{abstract}

Key Words: dopamine; serotonin; SPECT; midbrain; raphe nuclei

J Nucl Med 2015; 56:1036-1041

DOI: 10.2967/jnumed.115.153734

$\mathbf{P}$

arkinson disease (PD) is a neurodegenerative condition characterized by the progressive accumulation of Lewy body inclusions beginning from the medulla and olfactory nuclei and gradually spreading through the midbrain to the neocortex (1). In the human

Received Jan. 6, 2015; revision accepted Apr. 25, 2015.

For correspondence or reprints contact: Juho Joutsa, Turku PET Centre,

Turku University Hospital, P.O. Box 52, 20521 Turku, Finland.

E-mail: jtjout@utu.fi

Published online May 7, 2015.

COPYRIGHT (c) 2015 by the Society of Nuclear Medicine and Molecular Imaging, Inc. brain, there are 2 major dopamine systems. The nigrostriatal pathway originates from the substantia nigra (SN) pars compacta (region A9) projecting to the putamen, and the ventral dopamine pathways (mesolimbic and mesocortical pathways) originate from the ventral tegmental area (VTA, A10) and innervate the limbic system and neocortex. PD pathology shows regional selectivity within the SN affecting the most ventrolateral-tier neurons that mainly project to the dorsal putamen (2), and molecular in vivo imaging studies have shown corresponding posterior-to-anterior gradient in the degeneration of the dopamine function within the striatum (3). However, to the best of our knowledge, no prior studies have investigated the midbrain projections within the dopamine system in PD.

$\mathrm{PD}$ is primarily treated with medications that enhance the brain dopamine function, but not all symptoms of the disease respond favorably to the treatment. In addition, restoring the brain's dopamine function can lead to the development of behavioral disorders, such as impulse control disorders (ICDs) and psychotic symptoms. These disorders are linked with increased brain dopamine function, and they can appear even though the patient suffers from marked bradykinesia and rigidity from insufficient dopamine signaling in the nigrostriatal pathway (4-7). The hyperdopaminergic symptoms in medicated PD patients can be explained by the dopamine overdose hypothesis, which assumes a relative overactivation of the mesolimbic and mesocortical (referred later to as ventral), compared with nigrostriatal (referred to as dorsal), dopaminergic pathways $(8,9)$. As the degree of damage in the nigrostriatal pathway is more severe than in the mesocorticolimbic pathways, it might lead to a shift in the balance of mesencephalic projections from the dorsal striatum to limbic and frontal cortical regions (dorsal-to-ventral shift). Such a shift in the midbrain dopamine signaling would explain why even small doses of dopaminergic medications can induce behavioral side effects that are assumed to originate from the mesocorticolimbic pathways.

SPECT with the radioligand ${ }^{123}$ I-FP-CIT ( ${ }^{123} \mathrm{I}-\mathrm{N}-\omega$-fluoropropyl$2 \beta$-carbomethoxy-3 $\beta$-(4-iodophenyl)nortropane) is widely used in the differential diagnosis of PD. Several studies have provided uniform evidence that there is reduction in the striatal ${ }^{123}$ I-FP-CIT signal, which correlates with the severity of the nigrostriatal cell loss in PD (10). In the clinical practice, ${ }^{123}$ I-FP-CIT is used as a dopamine transporter (DAT) ligand, but it binds to other monoamine transporters as well. ${ }^{123}$ I-FP-CIT has the highest affinity for DAT, which is followed by the serotonin transporter (SERT), and low affinity for the norepinephrine transporter (11). Therefore, the 
neurotransmitter system under study depends on the relative density (and ligand affinity) of the monoamine transporters in the given brain region. For instance, in the dopamine-rich striatum ${ }^{123}$ I-FP-CIT binding can be considered specific for DAT, but the binding is mainly for SERT in the thalamus and raphe nuclei (12). However, the signal-to-noise ratio of ${ }^{123}$ I-FP-CIT in the extrastriatal regions is clearly lower than in the DAT-rich striatum, which has limited the extrastriatal use of ${ }^{123}$ I-FP-CIT. Also, the existing literature about monoaminergic function outside the striatum is nonuniform (13-17). Some of the studies have reported decreased serotonin function in PD, whereas other studies have provided inconclusive results or have been methodologically limited by a lack of reliable evaluation of the midbrain nuclei.

This study investigated a large group of PD $(n=216)$ and control patients $(n=204)$, enabling us to study the extrastriatal monoamine systems with high statistical power. The aim of the present study was to investigate the midbrain dopaminergic projections and to explore extrastriatal serotonergic abnormalities in PD that could be demonstrated with ${ }^{123}$ I-FP-CIT SPECT. We hypothesized that, in addition to the well-established degeneration of the nigrostriatal pathway, PD patients would show a dorsal-toventral shift in the functional dopaminergic midbrain projections. Moreover, we attempted to clarify possible abnormalities in the brain serotonin function particularly in the midbrain.

\section{MATERIALS AND METHODS}

The data were retrospectively collected from the patients of Turku University Hospital, as previously described (18). Of the 563 patients scanned with ${ }^{123}$ I-FP-CIT SPECT during 2007-2012, we identified 230 patients with normal scan results and no evidence of a neurodegenerative parkinsonism syndrome during the follow-up (control patients) and 231 patients who received a clinical diagnosis of PD after the follow-up and had an abnormal SPECT scan result consistent with the diagnosis (PD patients). Forty-one patients were excluded from the current study because of insufficient image quality (mainly inappropriate field of view excluding the midbrain from the image), leaving 204 control patients and 216 patients with PD for the final sample. The images were visually evaluated by nuclear medicine physician according to the current guidelines (19). The diagnoses were established after a clinical follow-up of $3.0 \pm 1.7$ y (mean $\pm \mathrm{SD}$; range, 0.3-6.1 y). The institutional review board approved this retrospective study, and the requirement to obtain informed consent was waived. The study was conducted according to the principles of the Declaration of Helsinki.

The subjects were scanned with 2 Infinia Hawkeye SPECT/CT scanners with 0.952-cm (0.375-in) crystals (GE Healthcare) $(n=156$ and 163) and a Picker Irix $\gamma$ camera with 1.90 -cm (0.75-in) crystals (Picker International) $(n=101)$. The subjects were instructed to discontinue all medications affecting the tracer binding before the tracer injection (19). The subjects received a rapid $185-\mathrm{MBq}$ bolus of ${ }^{123}$ I-FP-CIT and were scanned $4 \mathrm{~h}$ after the injection. One hundred twenty projections were acquired during $180^{\circ}$ of rotation $(64 \times 64$ voxels; zoom, 2.0 ; photopeak energy window, $159 \mathrm{keV} \pm 10 \%)$. The reconstruction was performed using the ordered-subsets expectation algorithm of Hybrid Recon Neurology software (version 1.0.15) including Chang's attenuation $(\mu=$ $0.146 \mathrm{1} / \mathrm{cm}$ ), collimator, and Monte Carlo-based scatter correction with 16 iterations and 4 subsets (Hermes Medical Solutions AB). A 3-dimensional gaussian postprocessing filter with $0.7 \mathrm{~cm}$ in full-width at halfmaximum was applied.

The image preprocessing was performed using Statistical Parametric Mapping software (SPM8; http://www.fil.ion.ucl.ac.uk/spm/ software/spm8/) running in Matlab R2011a (The MathWorks Inc.).
The spatial normalization of the SPECT images was conducted using an in-house ${ }^{123}$ I-FP-CIT SPECT template. The template was created using ${ }^{123}$ I-FP-CIT SPECT images from 11 subjects ( 5 with PD; 8 men; mean age $\pm \mathrm{SD}, 56 \pm 8.6 \mathrm{y}$ ) from this sample who also had excellent-quality (normal brain structure, appropriate field of view, 1-mm cubic voxels, and no motion artifacts) 3-dimensional T1-weighted 3-T MR images. The spatial normalization of the images of the template subjects was conducted using the anatomic information from the MR images (20). The specific binding ratio (SBR) images were calculated as the regionto-occipital cortex ratio and smoothed using a gaussian kernel of $8 \mathrm{~mm}$ in full width at half maximum.

The group differences were tested by applying a general linear model using the scanner; sex; age; and the symptom type, duration, and lateralization as nuisance covariates. Additionally, the effects of prior dopaminergic or antipsychotic medication use on SBR and other findings were separately tested by adding them to subsequent general linear model analyses. Analyses were restricted to the brain gray matter, pons, and midbrain (SPM WFU PickAtlas toolbox; http:// www.nitrc.org/projects/wfu_pickatlas/ (21)), which showed specific tracer binding (i.e., $\mathrm{SBR} \geq 1.0$ ) by creating a single analysis mask that was used in all analyses. The mean SBR extracted from the ventral midbrain cluster that showed group difference (cluster size, $904 \mathrm{~mm}^{3}$; peak at $-6-14-14 ; \mathrm{T}=6.78$, familywise error (FWE)corrected $P<0.000001$ ) was used as a variable of interest to investigate dopaminergic correlations between the midbrain and striatal and extrastriatal regions. A conservative FWE-corrected $P$ value of less than 0.05 at the voxel-level within the entire mask volume was considered statistically significant in all SPM analyses. The SBR values were extracted from statistically significant clusters with the Marsbar toolbox (http://marsbar.sourceforge.net/) to estimate the magnitude of the group differences and to illustrate the correlations. Clusters were overlaid on the T1-weighted MR image template using Mango software (version 2.6; http://ric.uthscsa.edu/mango). Individual-samples $t$ tests were used for the group comparisons, with mean cluster SBR values and demographic variables. In addition, PD patients with and without tremor were compared using $t$ tests to assess whether altered binding in SERT-rich regions was associated with motor symptom type. A $P$ value of less than 0.05 was set as the level of significance in all statistical analyses run in SPSS (version 21.0; IBM Corp.).

\section{RESULTS}

The study sample included 124 (57.4\%) PD men and 92 (50.5\%) control men $\left(\chi^{2}=2.02, P=0.16\right)$. PD patients were slightly older than controls (mean age $\pm \mathrm{SD}, 66.6 \pm 10.4 \mathrm{y}$ in PD vs. $63.9 \pm 12.2 \mathrm{y}$ in controls; $\mathrm{t}=2.45, P=0.02$ ). The mean PD motor symptom duration $\pm \mathrm{SD}$ at the time of SPECT scanning was $2.0 \pm 2.5 \mathrm{y}$, and $148(68.5 \%)$ of the patients had tremor. Only a few subjects had prior dopaminergic $(n=66,15.7 \%)$ or antipsychotic $(n=45$, $10.7 \%$ ) medication. Antipsychotic medication was more common in controls than in PD patients $(n=38$ [18.6\%] vs. 7 [3.2\%], $P<$ $0.001)$, but there were no differences in dopaminergic medication use between the groups ( $n=25[12.3 \%$ ] vs. 41 [19.0\%], $P=0.06)$.

PD patients had lower ${ }^{123}$ I-FP-CIT binding in the striatum (as expected based on the study design), ventral midbrain, and prefrontal cortex than controls (Fig. 1). Additionally, the effect extended to the bilateral insula and medial temporal lobe. ${ }^{123}$ IFP-CIT binding in the thalamus and raphe nuclei was bilaterally higher in PD patients than controls. The magnitudes of the group differences are presented in Figure 2. The presence of tremor was not associated with ${ }^{123}$ I-FP-CIT binding in the raphe nuclei $(\mathrm{t}=$ $0.56, P=0.58)$ or left $(\mathrm{t}=1.63, P=0.11)$ or right thalamus $(\mathrm{t}=$ $0.03, P=0.98)$ in PD patients. 


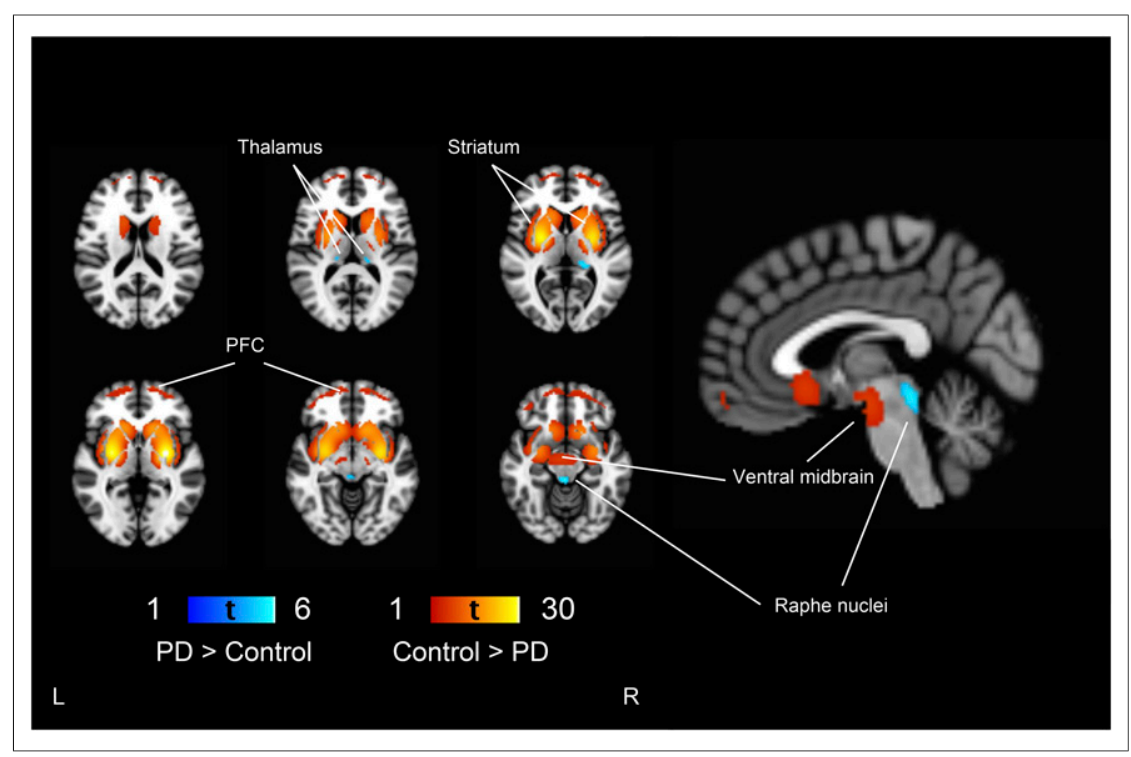

FIGURE 1. Group differences between control and PD patients. Regions showing lower ${ }^{123}$ I-FPCIT binding in PD patients are shown in red-yellow scale and higher binding in blue-light blue scale, compared with controls overlaid on brain T1 MR imaging template. Threshold is set to voxel-level FWE-corrected $P<0.05$ ( $\mathrm{t}>4.56)$. PFC $=$ prefrontal cortex.

In control patients, but not in PD patients, a positive correlation between the ventral midbrain and putamen ${ }^{123}$ I-FP-CIT binding was observed (Fig. 3). In PD patients, ventral midbrain binding correlated with the binding in the caudate, prefrontal cortex, insula, medial temporal lobe structures, anterior cingulate cortex, and posterior cingulate cortex, but this correlation was observed only in the very-anterior parts of the putamen (Fig. 3B). The extrastriatal correlations were clearly more restricted in controls (Fig. 3A). Scatterplots illustrating the correlations are presented in Figure 4.

A history of antipsychotic medication use was associated with higher, bilateral tracer binding in the head of the caudate nucleus in the entire sample, but it was not associated with higher binding in PD or control patients separately. A history of dopaminergic medication use did not have any effect on tracer binding. Furthermore, adding antipsychotic and dopaminergic medication use as nuisance covariates did not change the observed group differences or correlations.

\section{DISCUSSION}

The results of the present study show not only that PD is associated with loss of functional dopaminergic connections in the nigrostriatal pathway but also that the balance is shifted toward mesocorticolimbic
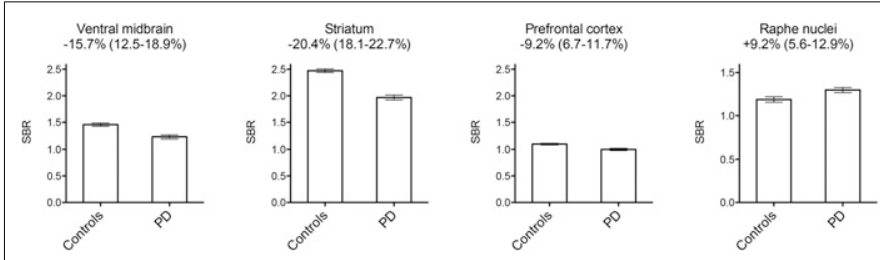

FIGURE 2. Regional dopamine and serotonin transporter density. Average specific binding ratios extracted from statistically significant clusters shown in Figure 1. Mean (95\% confidence interval) difference between Parkinson disease (PD) and control patients is given for each region. All group differences are significant at $P<0.001$. SBR $=$ specific binding ratio.

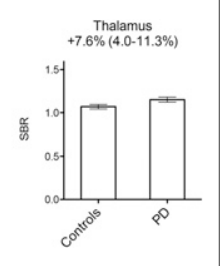

neurotransmission. In PD patients, ventral midbrain dopamine function correlates with multiple corticolimbic regions whereas the correlation is mainly limited to the putamen in subjects with normal dopamine function. This finding provides in vivo molecular evidence of the relative preservation of mesocorticolimbic dopamine neuron function as compared with nigrostriatal projections in PD. Furthermore, the present results show that ${ }^{123} \mathrm{I}$-FP-CIT binding is increased in serotonin-rich regions, suggesting that $\mathrm{PD}$ is associated with functional transporter upregulation in the serotonergic system in the early stages of the disease.

Previous molecular imaging studies have showed that PD is associated with substantial loss of striatal dopamine function $(3,10)$. The degree of dopaminergic damage is the most pronounced in the posterior parts of the striatum but also occurs in the anterior striatum and other brain regions (3). The present study corroborates previous findings in showing the loss of dopaminergic connection between the mesencephalon and posterior striatum. However, although many extrastriatal regions also show loss of DAT binding in PD, the dopaminergic correlation between mesencephalon and limbic/ cortical projection areas is in fact enhanced, indicating a shift from nigrostriatal to mesocorticolimbic dopaminergic projections (dorsalto-ventral shift).

The clinical sequelae of the shift from nigrostriatal to mesocorticolimbic dopaminergic projections in PD cannot be determined with the present data, but the findings are in agreement with the dopamine overdose hypothesis. The dopamine overdose hypothesis was initially suggested by Gotham, Brown, and Marsden based on their neuropsychologic experiments with levodopa challenge (8). They reported that PD patients were impaired in different cognitive tasks when on or off levodopa, which depended on the specific dopaminergic pathways activated by the task. The authors suggested that the findings could be explained by the differential state of dopamine signaling (i.e., under- or overactivation) of the involved pathway, which depends on the degree of the underlying degeneration of the pathway and its activation by medication. This hypothesis is widely acknowledged and has inspired decades of research (9). PD is associated with several symptoms that are not directly linked to the loss of nigrostriatal dopamine neurotransmission $(22,23)$. For instance, ICDs emerge after the initiation of dopaminergic medication, and they are linked to enhanced mesolimbic and mesocortical dopamine function $(4,5,7,24)$. The present study demonstrates that, in PD, there is a shift in the functional dopaminergic projections from the nigrostriatal to mesolimbic and mesocortical pathways. Because the effects of dopaminergic medications are dependent on number of functioning neurons, use of dopaminergic medication aimed to restore the loss of nigrostriatal dopamine might lead to a parallel overactivation of the 


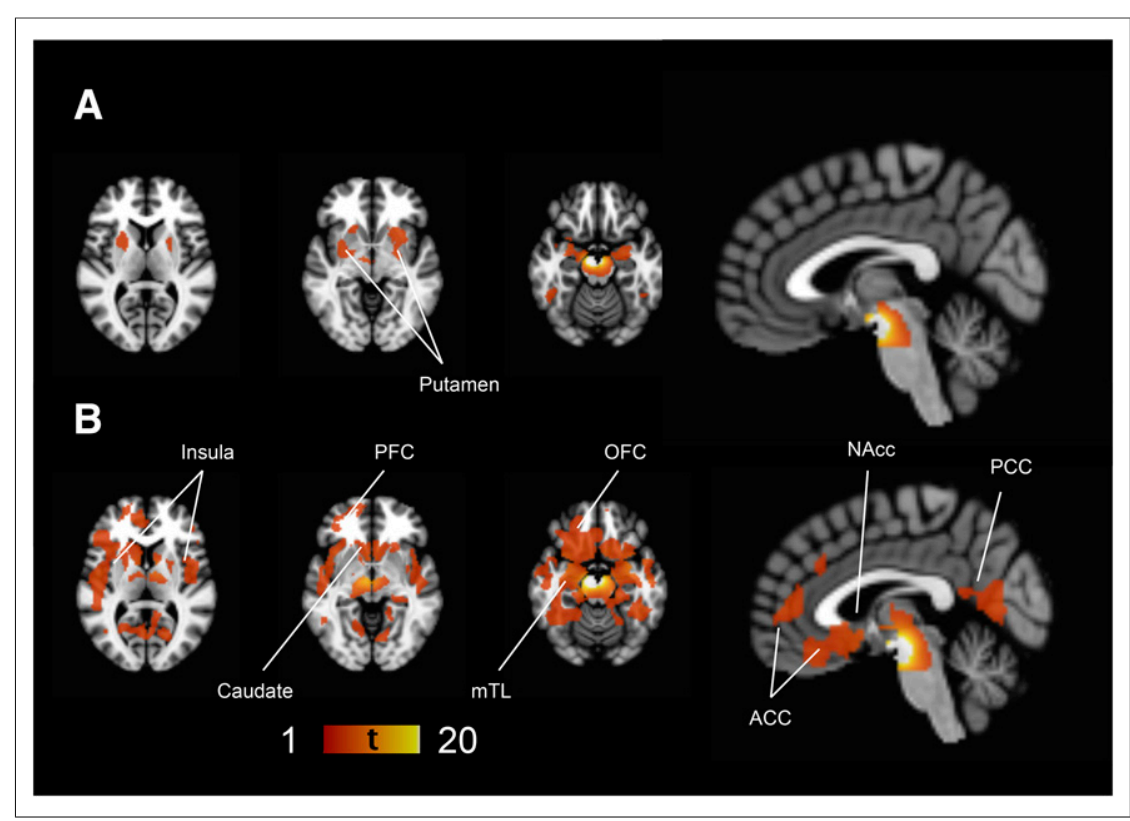

FIGURE 3. Ventral midbrain correlations. Regions showing statistically significant (voxel-level FWE-corrected $P<0.05$ corresponding to $t>4.81$ in control and $t>4.65$ in PD patients) correlations with ventral midbrain ${ }^{123}$-FP-CIT binding in controls (A) and PD patients (B). There is a strong autocorrelation in ventral midbrain area showing very high $T$ values that extends spatially to adjacent midbrain areas due to partial-volume effect. Left side of brain is on left in figure. ACC = anterior cingulate cortex; $\mathrm{mTL}=$ medial temporal cortex; NAcc $=$ nucleus accumbens area; OFC = orbitofrontal cortex; $\mathrm{PCC}=$ posterior cingulate cortex; $\mathrm{PFC}=$ prefrontal cortex.

relatively better preserved mesolimbic and mesocortical networks, resulting in the hyperdopaminergic state, possibly leading to the maladaptive behavioral change in some individuals (25). Although this interpretation needs to be corroborated in further imaging studies
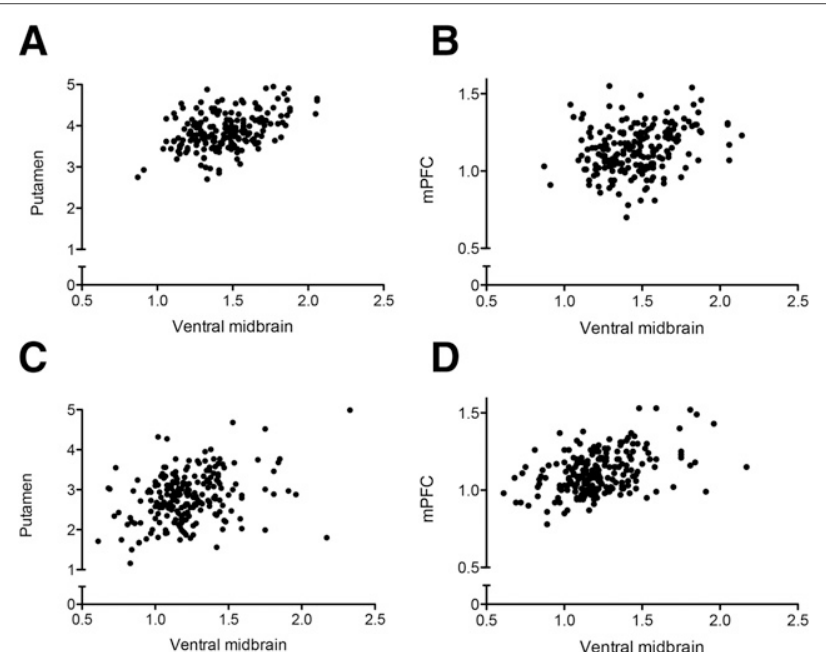

D

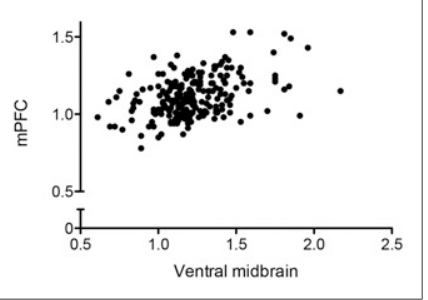

FIGURE 4. Scatterplots illustrating association between ventral midbrain and putamen or medial prefrontal cortex in controls ( $A$ and $B$ ) and PD patients (C and D). (A) Linear regression slope $y=0.97 x+2.48$, $r^{2}=0.23$. (B) $y=0.16 x+0.90, r^{2}=0.06$. (C) $y=0.89 x+1.75, r^{2}=0.12$. (D) $y=0.36 x+0.69, r^{2}=0.22$. Stronger associations exist between ventral midbrain and putamen in controls $(A)$, compared with $P D$ patients $(C)$, and between ventral midbrain and medial prefrontal cortex in PD patients (D), compared with controls (B). mPFC = medial prefrontal cortex. with patients with medication-related behavioral side effects, this view is supported by the recent study by Lee et al. reporting higher dopamine function in several extrastriatal regions, indicating relatively better preserved ventral dopamine pathways in PD-ICD patients, compared with PD patients without ICDs (24).

The ventral midbrain area includes both $\mathrm{SN}$ and VTA, and the signal from these very small structures cannot be reliably separated with the limited resolution of SPECT imaging. The observed enhanced dopaminergic midbrain correlation to the ventral striatum and extrastriatal regions in PD most likely reflects a higher proportion of the ventral midbrain signal originating from the VTA over SN neurons due to the excessive $\mathrm{SN}$ degeneration. However, the functional compensatory changes that increase the VTA neuron activity cannot be excluded. Furthermore, changes in nigrothalamic dopaminergic tract neurons may also be relevant in PD neurobiology (26), but because the ${ }^{123}$ I-FP-CIT binding in the thalamus is mainly from SERT, we were not able to investigate this issue with the present data.

We observed only a minor reduction of ${ }^{123}$ I-FP-CIT binding in the prefrontal cortical areas. This small reduction corresponds well with the neuropathologic progression of the disease because most of the patients are scanned at an early stage of the disease, likely corresponding to Braak stage III or IV, which is when the motor symptoms are evident but the clinical diagnosis might yet be unclear (27). At Braak stages III-IV, the Lewy body pathology covers the midbrain and basal ganglia area and begins to innervate the prefrontal regions, which corresponds well to the findings of the present study (27). Furthermore, age and the PD symptom duration, which might be linked with brain atrophy, were covaried in the analyses. We also found lower tracer binding in the insula and medial temporal lobe structures, but this probably reflects the partial-volume effect that spatially extends from the huge difference between the groups in the striatal binding.

According to the postmortem studies by Braak et al., serotonergic raphe nuclei are affected by the disease even before the dopaminergic neuron-containing regions. Earlier studies have provided inconclusive results about midbrain serotonin abnormalities in PD. Interestingly, studies have primarily reported decreased serotonin function, but no study has reported increases in the midbrain serotonin function, although there is substantial translational evidence suggesting enhanced serotonin function at some point in the course of the disease (28). For instance, some studies show compensatory changes, such as hyperinnervation of the serotonergic fibers, as well as an increase in synaptic serotonin concentration and neuronal firing rate in animal models of PD (29-31). Needless to say, there are also nonuniformities between the results in different studies with animal models of PD.

Politis et al. investigated $30 \mathrm{PD}$ patients and 10 controls with ${ }^{11} \mathrm{C}$-3-amino-4-(2-dimethylaminomethylphenylsulfanyl)-benzonitrile PET and reported evidence of decreased serotonin function that was progressive in parallel with the disease severity (32). 
However, raphe nuclei serotonin function was compromised only in the patients in the advanced group (disease duration over $10 \mathrm{y}$ ), compared with healthy volunteers. In the present study, we report increased raphe nuclei SERT density and similar changes in the thalamus in early PD patients. The symptom duration of the patients in the present study was an average of $2 \mathrm{y}$, and most of the patients $(84.3 \%)$ had no prior use of any antiparkinsonian medication. Therefore, even though the serotonin function is eventually compromised in $\mathrm{PD}$, there might be a compensatory upregulation in the serotonergic system comparable to the upregulation of striatal dopamine receptors in early PD $(33,34)$. Hence, we speculate that the degeneration of the serotonergic function becomes evident only after disease has progressed to a state in which compensatory mechanisms are no longer sufficient.

Tremor is a classic symptom of PD, but it is poorly explained via dopaminergic degeneration $(18,35)$. Several studies have suggested a role for reduced serotonin function in parkinsonian tremor, but the evidence remains inconclusive and trials with serotonergic medications have failed to alleviate tremor $(17,35,36)$. In the present study, we did not observe differences in the serotonin function between patients with and without tremor. It should be acknowledged that we compared patients with or without tremor and not patients with the tremor type or akinetic-rigid type of disease, which can diminish the differences. Alterations in the brain serotonin system have been speculated also to be important in several nonmotor symptoms of PD, such as depression, anxiety, fatigue, and sleep disturbances $(22,35)$. For instance, depressed PD patients have been shown to have higher midbrain SERT binding than nondepressed patients $(37,38)$. Furthermore, premotor symptoms of PD include depression, which could be related to the inappropriate upregulation of midbrain SERT that reduces the synaptic serotonin concentration early in the disease course (39).

Because the study population was collected retrospectively, clinical subtype and motor or nonmotor ratings (such as ICD or depression ratings) were not available for further correlation analyses. It cannot be excluded that our PD sample included cases of relatively rare atypical parkinsonism syndromes, which might have affected the results. However, all PD diagnoses were confirmed after an average 3-y follow-up by a neurologist, and the large sample size enabled us to also investigate the midbrain areas that have a relatively low signal-to-noise ratio. All effects were tested using a stringent statistical threshold (voxel-level FWE-corrected $P<0.05$ ). In addition, it cannot be excluded that the binding in cortical regions that correlates with ventral midbrain binding is partly from SERT. However, ${ }^{123}$ I-FP-CIT has clearly the highest affinity for DAT, and in striatal regions the signal is mainly from DAT $(11,12)$.

\section{CONCLUSION}

PD is associated with relatively enhanced dopaminergic projections to the limbic and cortical brain regions from the mesencephalon, which is consistent with the principles of the dopamine overdose hypothesis. Furthermore, the present results suggest that PD is associated with upregulated midbrain serotonin function at an early stage. The results provide insight into PD neurobiology, and further studies with parallel molecular imaging and clinical/behavioral testing are needed to clarify the significance of these findings.

\section{DISCLOSURE}

The costs of publication of this article were defrayed in part by the payment of page charges. Therefore, and solely to indicate this fact, this article is hereby marked "advertisement" in accordance with 18 USC section 1734. This work was supported by the Finnish Medical Foundation and the Turku University Hospital (EVO funds). Dr. Juho Joutsa has received a lecturer honoraria from Boehringer-Ingelheim and research grant from Lundbeck. Dr. Valtteri Kaasinen has received speaker honoraria or travel grants from Merck, Medtronic, Orion-Pharma, Abbvie, UCB, and Lundbeck and has served as a member of the advisory board of UCB and as a consultant for Orion-Pharma and Lundbeck. No other potential conflict of interest relevant to this article was reported.

\section{ACKNOWLEDGMENTS}

We are grateful for the staff of department clinical physiology and nuclear medicine for their skilled work during the imaging.

\section{REFERENCES}

1. Braak H, Del Tredici K, Rüb U, de Vos RA, Jansen Steur EN, Braak E. Staging of brain pathology related to sporadic Parkinson's disease. Neurobiol Aging. 2003;24:197-211.

2. Fearnley JM, Lees AJ. Ageing and Parkinson's disease: substantia nigra regional selectivity. Brain. 1991;114:2283-2301.

3. Nandhagopal R, Kuramoto L, Schulzer M, et al. Longitudinal progression of sporadic Parkinson's disease: a multi-tracer positron emission tomography study. Brain. 2009;132:2970-2979.

4. Weintraub D, Koester J, Potenza M, et al. Impulse control disorders in Parkinson disease: a cross-sectional study of 3090 patients. Arch Neurol. 2010;67:589-595.

5. Steeves TD, Miyasaki J, Zurowski M, et al. Increased striatal dopamine release in Parkinsonian patients with pathological gambling: a $\left[{ }^{11} \mathrm{C}\right]$ raclopride PET study. Brain. 2009;132:1376-1385.

6. O'Sullivan SS, Wu K, Politis M, et al. Cue-induced striatal dopamine release in Parkinson's disease-associated impulsive-compulsive behaviours. Brain. 2011;134:969978.

7. Joutsa J, Martikainen K, Niemelä S, et al. Increased medial orbitofrontal $\left[{ }^{18} \mathrm{~F}\right]$ fluorodopa uptake in Parkinsonian impulse control disorders. Mov Disord. 2012;27:778-782.

8. Gotham AM, Brown RG, Marsden CD. 'Frontal' cognitive function in patients with Parkinson's disease 'on' and 'off' levodopa. Brain. 1988;111:299-321.

9. Vaillancourt DE, Schonfeld D, Kwak Y, Bohnen NI, Seidler R. Dopamine overdose hypothesis: evidence and clinical implications. Mov Disord. 2013;28:19201929.

10. Kraemmer J, Kovacs GG, Perju-Dumbrava L, Pirker S, Traub-Weidinger T, Pirker W. Correlation of striatal dopamine transporter imaging with post mortem substantia nigra cell counts. Mov Disord. 2014;29:1767-1773.

11. Scheffel U, Lever JR, Abraham P, et al. N-substituted phenyltropanes as in vivo binding ligands for rapid imaging studies of the dopamine transporter. Synapse. 1997;25:345-349.

12. Koch W, Unterrainer M, Xiong G, et al. Extrastriatal binding of $\left[{ }^{123} \mathrm{I}\right]$ FP-CIT in the thalamus and pons: gender and age dependencies assessed in a European multicentre database of healthy controls. Eur J Nucl Med Mol Imaging. 2014;41: 1938-1946.

13. Kim SE, Choi JY, Choe YS, Choi Y, Lee WY. Serotonin transporters in the midbrain of Parkinson's disease patients: a study with ${ }^{123}$ I-beta-CIT SPECT. $J$ Nucl Med. 2003;44:870-876.

14. Isaias IU, Marzegan A, Pezzoli G, et al. A role for locus coeruleus in Parkinson tremor. Front Hum Neurosci. 2011;5:179.

15. Isaias IU, Marotta G, Pezzoli G, et al. Enhanced catecholamine transporter binding in the locus coeruleus of patients with early Parkinson disease. BMC Neurol. 2011;11:88.

16. Roselli F, Pisciotta NM, Pennelli M, et al. Midbrain SERT in degenerative parkinsonisms: a ${ }^{123}$ I-FP-CIT SPECT study. Mov Disord. 2010;25:1853-1859.

17. Caretti V, Stoffers D, Winogrodzka A, et al. Loss of thalamic serotonin transporters in early drug-naïve Parkinson's disease patients is associated with tremor: an [ $\left.{ }^{123} \mathrm{I}\right] \beta$-CIT SPECT study. J Neural Transm. 2008;115:721-729.

18. Kaasinen V, Kinos M, Joutsa J, Seppänen M, Noponen T. Differences in striatal dopamine transporter density between tremor dominant and non-tremor Parkinson's disease. Eur J Nucl Med Mol Imaging. 2014;41:1931-1937. 
19. Darcourt J, Booij J, Tatsch K, et al. EANM procedure guidelines for brain neurotransmission SPECT using ${ }^{123}$ I-labelled dopamine transporter ligands, version 2. Eur J Nucl Med Mol Imaging. 2010;37:443-450.

20. Ashburner J. A fast diffeomorphic image registration algorithm. Neuroimage. 2007;38:95-113.

21. Maldjian JA, Laurienti PJ, Kraft RA, Burdette JH. An automated method for neuroanatomic and cytoarchitectonic atlas-based interrogation of fMRI data sets. Neuroimage. 2003;19:1233-1239.

22. de la Riva P, Smith K, Xie SX, Weintraub D. Course of psychiatric symptoms and global cognition in early Parkinson disease. Neurology. 2014;83:1096-1103.

23. Todorova A, Jenner P, Ray Chaudhuri K. Non-motor Parkinson's: integral to motor Parkinson's, yet often neglected. Pract Neurol. 2014;14:310-322.

24. Lee JY, Seo SH, Kim YK, et al. Extrastriatal dopaminergic changes in Parkinson's disease patients with impulse control disorders. J Neurol Neurosurg Psychiatry. 2014;85:23-30.

25. Cilia R, van Eimeren T. Impulse control disorders in Parkinson's disease: seeking a roadmap toward a better understanding. Brain Struct Funct. 2011;216:289-299.

26. García-Cabezas MA, Rico B, Sánchez-González MA, Cavada C. Distribution of the dopamine innervation in the macaque and human thalamus. Neuroimage. 2007;34:965-984.

27. Hawkes CH, Del Tredici K, Braak H. A timeline for Parkinson's disease. Parkinsonism Relat Disord. 2010;16:79-84.

28. Miguelez C, Morera-Herreras T, Torrecilla M, Ruiz-Ortega JA, Ugedo L. Interaction between the 5-HT system and the basal ganglia: functional implication and therapeutic perspective in Parkinson's disease. Front Neural Circuits. 2014; $8: 21$.

29. Balcioglu A, Zhang K, Tarazi FI. Dopamine depletion abolishes apomorphine- and amphetamine-induced increases in extracellular serotonin levels in the striatum of conscious rats: a microdialysis study. Neuroscience. 2003;119:1045-1053.
30. Rozas G, Liste I, Guerra MJ, Labandeira-Garcia JL. Sprouting of the serotonergic afferents into striatum after selective lesion of the dopaminergic system by MPTP in adult mice. Neurosci Lett. 1998;245:151-154.

31. Prinz A, Selesnew LM, Liss B, Roeper J, Carlsson T. Increased excitability in serotonin neurons in the dorsal raphe nucleus in the 6-OHDA mouse model of Parkinson's disease. Exp Neurol. 2013;248:236-245.

32. Politis M, Wu K, Loane C, et al. Staging of serotonergic dysfunction in Parkinson's disease: an in vivo ${ }^{11}$ C-DASB PET study. Neurobiol Dis. 2010;40:216-221.

33. Kaasinen V, Ruottinen HM, Någren K, Lehikoinen P, Oikonen V, Rinne JO. Upregulation of putaminal dopamine D2 receptors in early Parkinson's disease: a comparative PET study with $\left[{ }^{11} \mathrm{C}\right]$ raclopride and $\left[{ }^{11} \mathrm{C}\right] N$-methylspiperone. $J$ Nucl Med. 2000;41:65-70.

34. Rinne UK, Laihinen A, Rinne JO, Någren K, Bergman J, Ruotsalainen U. Positron emission tomography demonstrates dopamine D2 receptor supersensitivity in the striatum of patients with early Parkinson's disease. Mov Disord. 1990; 5:55-59.

35. Politis M, Niccolini F. Serotonin in Parkinson's disease. Behav Brain Res. 2015;277:136-145.

36. Huot $\mathrm{P}$, Fox SH. The serotonergic system in motor and non-motor manifestations of Parkinson's disease. Exp Brain Res. 2013;230:463-476.

37. Boileau I, Warsh JJ, Guttman M, et al. Elevated serotonin transporter binding in depressed patients with Parkinson's disease: a preliminary PET study with $\left[{ }^{11} \mathrm{C}\right]$ DASB. Mov Disord. 2008;23:1776-1780.

38. Politis M, Wu K, Loane C, et al. Depressive symptoms in PD correlate with higher 5-HTT binding in raphe and limbic structures. Neurology. 2010;75:19201927.

39. Leentjens AF, Van den Akker M, Metsemakers JF, Lousberg R, Verhey FR. Higher incidence of depression preceding the onset of Parkinson's disease: a register study. Mov Disord. 2003;18:414-418. 